\title{
Effect of Dispersion Medium and Dominated Evaporation On the Pattern Formation of Colloidal Suspension
}

\author{
Wenjie Ma ${ }^{1, a}$, Yuren Wang ${ }^{2}$ \\ ${ }^{1}$ College of Science, Northeast Dianli University, Jilin 132012, China \\ ${ }^{2}$ National Microgravity Laboratary, Institute of Mechanics, Chinese Academy of Sciences, Beijing \\ 100190, China \\ amawenjie81@gmail.com
}

Keywords: Dispersion medium; Marangoni convection; Broad-ring pattern.

\begin{abstract}
We study the effect of dispersion medium and dominated evaporation on the drying process and pattern of thin liquid layer colloidal suspention. Panasonic camera is used to capture the drying process and macroscopic pattern. It is shown that drying patterns are sensitive to the dispersion medium. As the volume ratio of ethanol in aqueous ethanol colloidal suspension increases, the evaporation rate increases, and film in the center becomes smaller and thinner, obvious broad-ring pattern can be observed. We suggest that difference in dispersion medium changes the evaporation rate, which has a direct proportional variation with strength of Marangoni convection. Furthermore, convection strength has great influence on self-assembly of colloid particles and the pattern formation of colloidal suspension. We design two simple experiments to change the evaporation rate and convection strength in order to verify the conclusion above. Broad-ring patterns with spray-like round edge at higher evaporation rate are obtained. Uniform film forms at lower evaporation rate.
\end{abstract}

\section{Introduction}

When a suspension droplet dries on a glass surface, particles accumulate near the edge of the droplet boundary and often leave a ring-like pattern as the droplet evaporates. Formation of ring-like pattern is a general phenomenon such as the residue left when coffee drying on the countertop and mineral rings left on washed glass ware. Controlling the distribution of solute during drying plays an important role in industry and our daily life. For example, paint manufactures use a variety of additives to ensure that the pigment is evenly dispersed during drying [1]. Generally speaking, most drying patterns are dominated by the dissipation of free energy and the self-organization of finely granular matter in open systems. Since patterns themselves in nature are complex to study, great attention has been devoted to the dissipative structural pattern formation in the drying process of colloidal suspension. According to the literatures [2-6], quite similar broad-ring patterns are observed in despite of kinds of suspensions and substrates. Electrostatic interparticle interactions have been pointed out as one of the important factors for the dissipative structures. Hydrophobic and hydrophilic interactions are also demonstrated to be important during the drying process. In addition, the role of capillary force and convection flow in the dissipative pattern are also emphasized. Since a large-scale nanostructured film with a flat and uniform surface is anticipated for applications in photonic crystal engineering, it is necessary to avoid broad-ring formation. Deeply understanding the drying process and influence factor of colloidal suspension should be of great importance to those attempting to harness this phenomenon.

In this study, the drying processes and patterns of colloidal suspensions in which silica spheres dispersed in ethanol, water and mixtures of ethanol and water were studied in open containers at room temperature. In addition, two simple experiments were designed to verify the influence of evaporation rate on the drying macroscopic pattern of colloidal suspensions. 


\section{Experimental}

The diameter of silica colloidal particle is $450 \mathrm{~nm}$, and the relative deviation in size is less than $5 \%$. The silica spheres were respectively dispersed into water, ethanol and the mixtures of water and ethanol with the assistance of an ultrasound bath forming $0.36 \mathrm{vol} . \%$ suspensions. Volume fraction of ethanol are 0,20,60,100vol.\% repectively. The glass substrates were rinsed with deionized water and dried under a flow of nitrogen gas. For each sample, a circular silica glue mat with $1 \mathrm{~cm}$ inner diameter was set onto a glass substrate, and $180 \mu \mathrm{l}$ colloidal suspension was dropped onto the glass substrate. The samples were placed on a desk until dried up completely in a room air-conditioned at $22^{\circ} \mathrm{C}$. In order to verify the effect of the evaporation rate on the pattern formation of colloidal suspension, the colloidal suspensions of $0.36 \mathrm{vol} . \%$ and $1.36 \mathrm{vol} . \%$ were dried at $50^{\circ} \mathrm{C}$ in the temperature humidity chamber. In addition, One glass sheet with a hole in the central part was placed on silica glue mat to decrease the evaporation rate. Drying processes and macroscopic patterns of colloidal silica spheres were captured with a Panasonic digital camera.

\section{Results and Discussion}

Fig. 1 shows the macroscopic sedimention and drying patterns of silica colloidal suspensions in open containers at $22^{\circ} \mathrm{C}$. Volume ratio of ethanol in dispersion medium is respectively $0 \%, 20 \%, 60 \%$, $100 \mathrm{vol} \%$. It is found that drying patterns are sensitive to the ehanol content in the colloidal suspensions. Fig. 1(a) shows the drying pattern of colloid silica spheres dispersed in purely water. We find that flat film forms in the center and rough drying points distribute at the edge of the pattern. Fig. 1 (b) and (c) show that with the increase of the ethanol content in the colloidal suspension, film in the center becomes smaller and thinner, and a few ring-like patterns distribute at the outer edge. When colloid silica spheres are dispersed completely in purely ethanol, very obvious multiple broad-ring pattern with a void in the center is observed, as shown in Fig. 1(d).

Self-assembly of colloidal particles is dominated by sedimentation and convection during the drying process of colloidal suspension. Sedimentation itself cannot affect the radial distribution of colloidal particles and influence the formation of various patterns. In our experiment, since the liquid layer is very thin, the gravitational convection can be left out. Therefore, the dissipative pattern forms mainly under the influence of Marangoni convection. For an open container, the dispersion medium vapour directly evaporates from the surface of colloidal suspension. The rate of evaporation at the edge is greater than that at the centre. To compensate for dispersion medium loss at the edge, the capillary flow occurs from the centre to the edge, enabling colloidal partilces to flow and accumulate near the round edge. In addition, we noticed that strength of Marangoni convection can be evaluated by the evaporation rate. According to a Peclet number Pe, proposed by Routh and Russel, defined as

$$
\mathrm{Pe}=\mathrm{HE} / \mathrm{D}_{0}
$$

Where $\mathrm{H}$ is the initial thickness of the film, $\mathrm{E}$ the rate of evaporation, D0 the diffusion coefficient of the colloids[7]. The Peclet number is used to denote relative proportion of convection and diffusion. From the above equation, it is easily to be understood that convection strength has a proportional variation with the evaporation rate. For our experiment, distinct difference is observed among the drying patterns of the suspensions containing different ethanol content. One main reason is that different boiling points of ethanol and water lead to different evaporation rate. Boiling points of ethanol and water are respectively $78^{\circ} \mathrm{C}$ and $100^{\circ} \mathrm{C}$. Lower boiling point is, higher evaporation rate is. As shown in Fig. 1(a) and (b), when colloidal suspensions contain less amount of ethanol, the evaporation rate is lower and convection strength is weaker, most colloid particles distribute in the center after drying. With the increase of ethanol content in dispersion medium, the evaporation rate increases, therefore strength of Marangoni convection increases and multiple broad-ring patttern is observed, as can be seen from Fig. 1(c) and (d). 


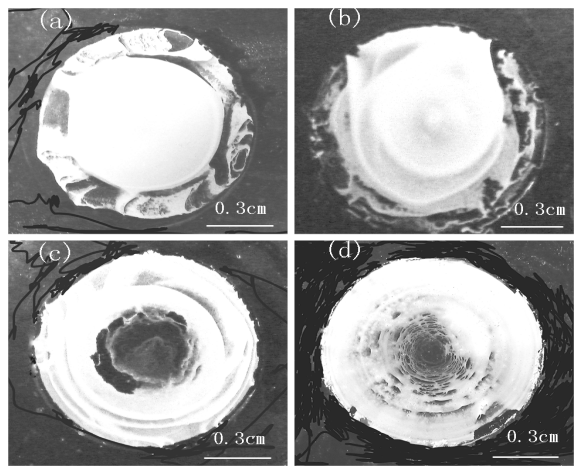

Fig. 1 Drying patterns of colloidal suspensions with different ethanol content in open containers at $22{ }^{\circ} \mathrm{C}(\varphi=0.0036)(\mathrm{a})[\mathrm{EtOH}] /[\mathrm{H} 2 \mathrm{O}]=0: 1$ (b) $1: 4$ (c) $3: 2$ (d) $1: 0$

Fig. 2 shows drying processes for colloidal suspensions containing different ethanol content ranging from $0 \%$ to $100 \mathrm{vol} . \%$. The T-values are $13.25 \mathrm{~h}, 6 \mathrm{~h}, 3.75 \mathrm{~h}$, and $1.5 \mathrm{~h}$ after the suspensions dried up completely for $0,20,60,100 \mathrm{vol} . \%$ of ethanol, respectively. T decreases substantially as ethanol content increases. As shown in Fig. 2, with the increase of ethanol content in dispersion medium, drying time decreases, and more obvious and intense convection phenomenon is observered. When the dispersion medium is purely water(Fig. 2(a-c)), the evaporation rate is relatively low, and convection strength is very weak. Most colloid particles sediment under the influence of gravity, and less amount of colloid particles flow from the center to the edge. Thus, flat film forms in the center and few drying points distribute at the edge of the pattern. When the dispersion medium is purely ethanol(Fig. 2(j-1)), the evaporation rate is relatively higher, and convection strength is more vigorous, broad-ring pattern with void in the central part forms. In addition, it is worth noting that when the dispersion medium is purely water and mixtures of ethanol and water, ring-like liquid layers near the round edge occur rupturing suddenly during drying process, which has certain influence on the final drying pattern, as can be seen from Fig. 2(b,e,h). This phenomenon is related to interface instability. When liquid layer is very thin(10-100nm), even in static liquid layer, rupture occurs because of long-range molecular force.

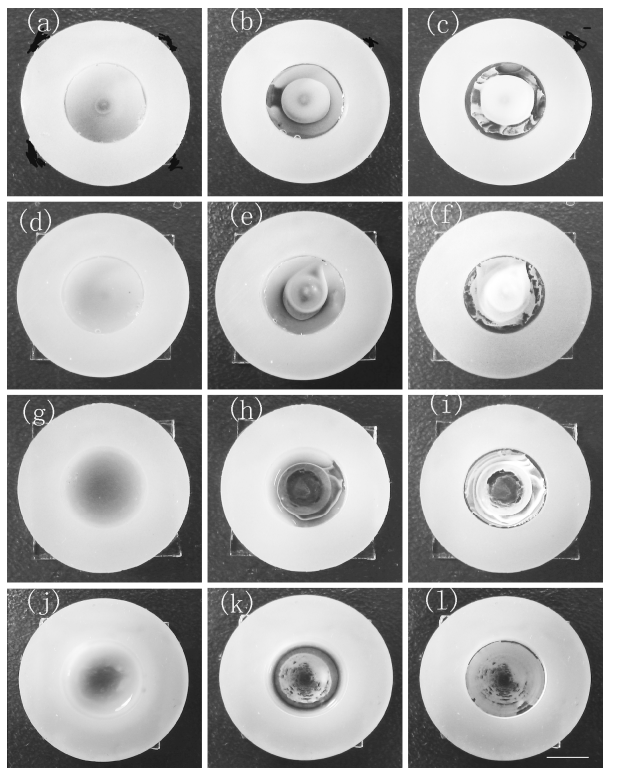

Fig. 2 Drying process of colloidal suspensions with different ethanol content $(\varphi=0.0036)$ (a-c) EtOH:H2O=0:1 (d-f) 1:4 (g-i) 3:2 (j-1) 1:0(a)After 9h (b) 11.5h (c) 13.25h (d) 4h (e) 5.25h (f) 6h (g) $1.5 \mathrm{~h} \mathrm{(h)} 2.45 \mathrm{~h} \mathrm{(i)} 3.75 \mathrm{~h} \mathrm{(j)} 0.75 \mathrm{~h} \mathrm{(k)} 1.25 \mathrm{~h} \mathrm{(l)} 1.5 \mathrm{~h}$ 
According to the results and discussion above, since the evaporation rate can influence the convection strength and drying pattern, we can change the drying pattern and produce more homogeneous film by dominating the evaporation rate. As is well known, the evaporation rate is a function of temperature, humidity and pressure. In several studies of evaporating liquid layers, the mass flux $\mathrm{J}$ is assumed to obey the Hertz-Knudsen equation, derived from the kinetic theory of gas[8-10]. The non-equilibrium law is derived for a flat interface along which the equality of liquid and vapour temperature is assumed (the vapour is further assumed to be a perfect gas), and reads

$$
J=\beta \sqrt{\frac{M}{2 \pi R T}}\left[P_{s}(T)-P_{0}(T)\right] .
$$

Where $\beta$ is the accommodation coefficient, $M$ is the molecular weight of vapour, $\operatorname{Ps}(T)$ is the saturation pressure at surface temperature $\mathrm{T}, \mathrm{P} 0(\mathrm{~T})$ is the vapour pressure just beyond the interface, $\mathrm{P} 0(\mathrm{~T})=\operatorname{Ps}(\mathrm{T}) \varphi$, $\varphi$ is the relative humidity. Obviously, the changes of temperature and relative humidity can lead to change of the evaporation rate finally.

Fig. 3 shows drying patterns of colloidal suspensions in open containers at higer evaporation temperature of $50^{\circ} \mathrm{C}$, in which colloid silica spheres are dispersed in purely ethanol. The volume fractions of colloid spheres are respectively 0.0036 and 0.0136 . It is found that when the colloidal suspensions are dried up at higher temperature, broad-ring patterns with spray-like round edge appear. Compared Fig. 3(a) with Fig. 1(a), increase of temperature leads to increase of the evaporation rate, which can influence the convection strength, therefore different patterns after drying are obtained.
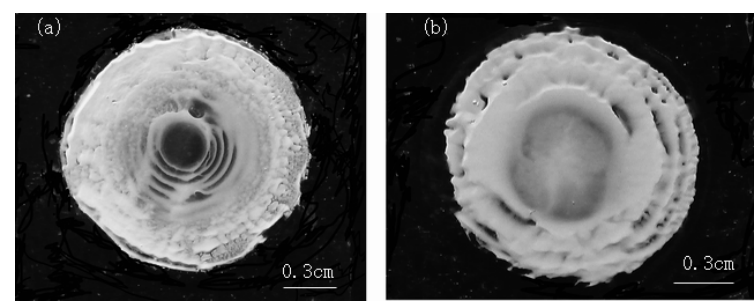

Fig. 3 Drying patterns formed for colloidal suspensions in open containers at $50{ }^{\circ} \mathrm{C}, 100 \mathrm{vol} . \%$ $\operatorname{EtoH}(\mathrm{a}) \varphi=0.0036$ (b) $\varphi=0.0136$

Fig. 4 shows the drying process and final pattern of colloidal suspension in a container covering a glass sheet with one hole in the centeral part. Apparently, the time for dryness is shortened by adding a lid, and the evaporation rate can be reduced. Homogeneous film is produced at lower evaporation rate and weaker convection strength.

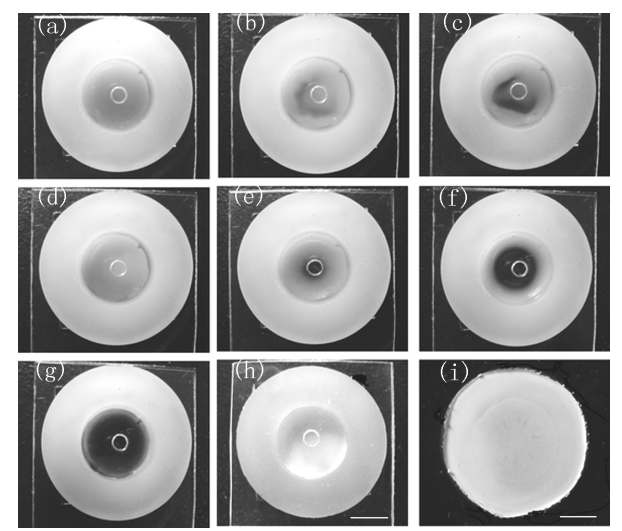

Fig. 4 Drying process of colloidal suspension in a container covering a lid with one hole in the central part(100 vol.\% EtoH, $\varphi=0.0036$ ) (a)0h (b) $1 \mathrm{~h}$ (c) $2 \mathrm{~h}$ (d)3h (e) $7.5 \mathrm{~h}$ (f) $9 \mathrm{~h} \mathrm{(g)10h} \mathrm{(h)} \mathrm{11h,} \mathrm{dry} \mathrm{up}$ (i) final pattern 


\section{Conclusions}

In summary, our experiments show that patterns of the dried films are sensitive to the ethanol content in the aqueous ethanol colloidal suspensions. We make clear that difference in dispersion medium changes the evaporation rate, which has proportional variation with convection strength. Convection strength has great influence on self-assembly of colloid particles and the pattern formation of colloidal suspension. We design two simple experiments to change the drying pattern by dominating the evaporation rate and convection strength. Increase of temperature results in the increase of the evaporation rate, broad-ring patterns with spray-like round edge are observed. Adding a lid with one hole on the drying container can reduce the evaporation rate, and homogeneous film is produced.

\section{Acknowledgment}

This work is supported by the Doctoral Fundation of Northeast Dianli University (Grant No. BSJXM-200913)

\section{References}

[1] R. D. Deegan, O. Bakajin, T. F. Dupont and G. Huber: Phy. Rev. E Vol. 62 (2000), p.756

[2] T. Okubo, S. Okuda and H. Kimura: Colloid Polym. Sci. Vol. 280 (2002), p.454

[3] T. Okubo, S. Okuda, H. Kimura and A. Tsuchida: Colloid Polym. Sci. Vol. 284 (2006), p.396

[4] T. Okubo, S. Kanayama, H. Ogawa and M. Hibino: Colloid Polym. Sci. Vol. 282 (2004), p.230

[5] T. Okubo, C. Shinoda, K. Kimura and A. Tsuchida: Langmuir Vol. 21 (2005), p.9889

[6] T. Yamaguchi, K. Kimura, A. Tsuchida and T. Okubo: Colloid Polym. Sci. Vol. 283 (2005), p.1123

[7] A. F. Routh and W. B Russel: Langmuir Vol.15 (1999), p.7762

[8] F. J Higuera: Phys. Flulid Vol. 30 (1987), p.679

[9] A. Prosperetti and M. S. Plesset: Phys. Flulid Vol. 27 (1984), p.1590

[10] H. J. Palmer: J. Fluid Mech. Vol. 75 (1976), p.487 
Machinery, Materials Science and Engineering Applications, MMSE2011

10.4028/www.scientific.net/AMR.228-229

Effect of Dispersion Medium and Dominated Evaporation on the Pattern Formation of Colloidal Suspension

10.4028/www.scientific.net/AMR.228-229.732 